\title{
THE EFFECT OF HYDROGEN CHARGING ON THE QUALITY OF ZINC AND POWDER PAINT COATINGS
}

\author{
${ }^{1}$ Kateřina KREISLOVÁ, ${ }^{1}$ Libor TUREK, ${ }^{1}$ Lubomír MINDOŠ \\ 1SVÚOM Ltd., Prague, Czech Republic, EU, kreislova@svuom.cz
}

https://doi.org/10.37904/metal.2020.3544

\begin{abstract}
There are shown some case studies when the defects in coatings (hot dip galvanised and duplex coatings with powder paints) were caused by hydrogen charging into matrix of substrate steel. Steel was exposed into hydrochloric acid and then the zinc coatings had been formed at higher temperature. The evidence of hydrogen recombination and its molecules' diffusion from coating layers is shown on cross-sections. The hydrogen release caused the defects on subsequently powder coated galvanized plates, too.
\end{abstract}

Keywords: Steel, galvanized, hydrogen charging, coating defects, microscope cross-section

\section{INTRODUCTION}

During hydrogen embrittlement, hydrogen is introduced to the surface of a metal and individual hydrogen atoms diffuse through the metal structure. Adsorbed hydrogen species recombine to form hydrogen molecules, creating pressure from within the metal. Hydrogen embrittlement can occur during various manufacturing operations or operational use - anywhere that the metal comes into contact with atomic or molecular hydrogen [1]. At room temperature, hydrogen atoms can be absorbed by carbon steel alloys. The absorbed hydrogen may be present either as atomic or molecular form. Processes that can lead to this include some technologies of surface treatment as phosphating, acidic pickling, and electroplating. Given enough time, the hydrogen diffuses to the metal grain boundaries and forms bubbles at the metal grain boundaries. These bubbles exert pressure on the metal grains. The trapped hydrogen atoms have high tendency to recombination to molecules with increased pressure (Figure 1). Despite a large research effort in attempting to understand the mechanisms of failure and in developing potential mitigating solutions, hydrogen embrittlement mechanisms are still not completely understood.
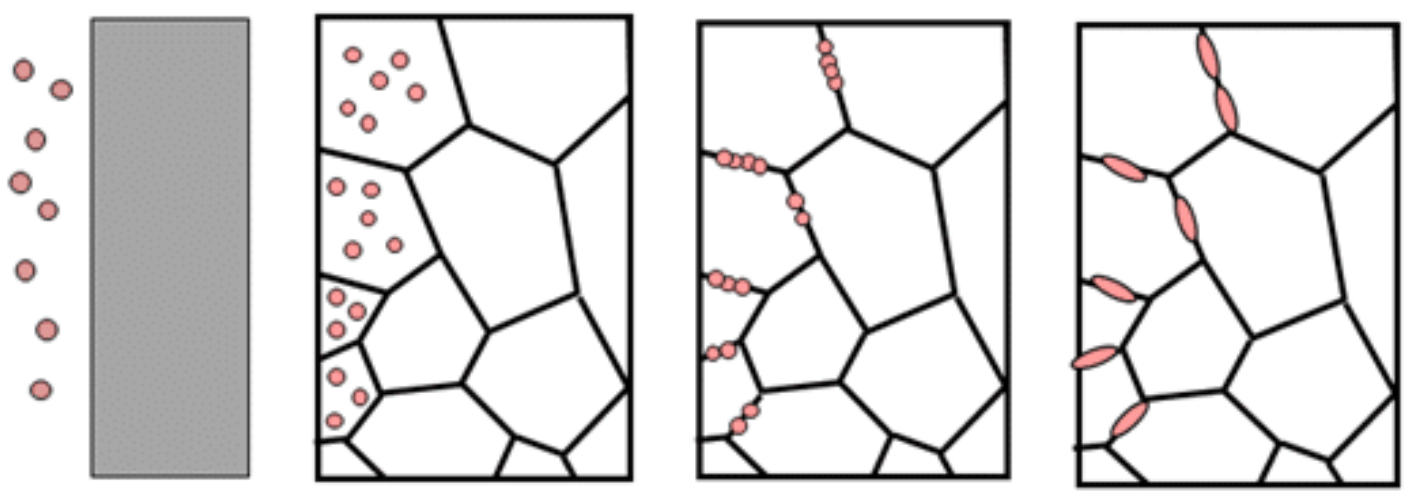

Figure 1 Mechanisms of hydrogen diffusion and recombination

Some types of steel and fabrication techniques that require extreme cold-working of the steel before galvanizing can lead to embrittlement problems that impact the performance of the product. During 
galvanization, hydrogen may be absorbed in the steel during the course of the pickling process, as a result of contact with the hydrogen ions present in the hydrochloric acid. Hydrochloric acid $(\mathrm{HCl})$ is used at $\mathrm{ca} 30^{\circ} \mathrm{C}$ temperature in hot-dip galvanizing processes, almost entirely for pickling prior to galvanizing. The concentration of acid is generally $10 \%-15 \% \mathrm{HCl}$. In next technological step as a result of the high temperature of the zinc bath the hydrogen atoms rapidly recombined. Most of the hydrogen is desorbed below $280^{\circ} \mathrm{C}$.

The two case studies presented in this paper concern defects and causes of failure of surface treatment associated with galvanized steel sheet material.

\section{CASE STUDIES}

\subsection{Small articles galvanized by centrifuge process}

One of the case studies presents defects associated with the actual hot-dip galvanizing process itself. The small articles (Figure 2) made from hot-rolled steel sheet had been galvanized by process known as centrifuge (or spin) galvanizing. In centrifuge galvanizing the articles are loaded into a basket which is spun at high speed immediately after removal from the molten zinc. Centrifuge galvanizing was conducted by high temperature process at about $550^{\circ} \mathrm{C}$. In the high temperature galvanizing process produces a coating comprised fully of zinc-iron alloy. Occasionally small centrifuged articles which have been cold-pressed or cold-drawn may exhibit some reduction in adherence of the zinc coating to the steel surface.

The low zinc coating adhesion was the problem with tested articles - sample 1. As the hot-rolled steel strips had been covered by thick layer of mild scale the strips had been pickled in hydrochloric acid before articles' forming and once again before galvanizing. The same articles had been made from cold-rolled steel without layer of mild scale - sample 2. On these articles the zinc layer shows low adhesion, too. Adhesion was tested by device for adhesive strength tests compliant with ASTM A123 (removed in 1989 edition). As reference sample was used very similar articles galvanized in the same conditions - sample 3 , which zinc layer was adherent.
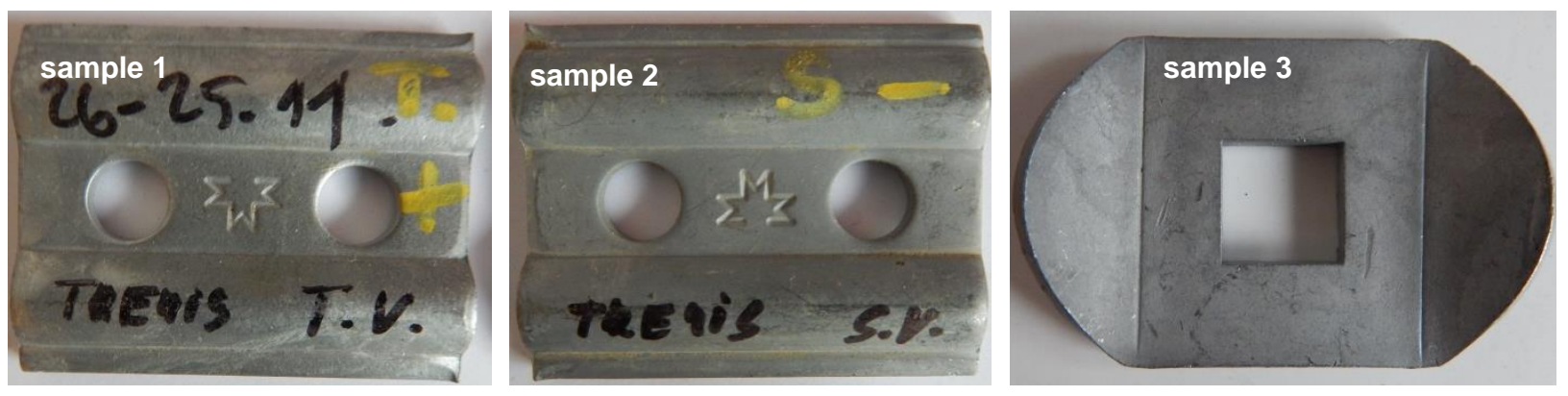

Figure 2 The galvanized articles - samples

The chemical composition of substrate steel was analyzed by EDX method on electron scanning microscope Tescan Vega II with detector X-Max 50 SDD, Oxford Instruments. In Table 1 there are given the elements significant for galvanizing. The composition steels are practically the same - category $\mathrm{C}(>0,03 \% \mathrm{Si}$ to $<0,14$ $\%$ Si) according to ISO 14712-2, i.e. not better steel for galvanizing.

The electron scanning microscope was used for SEM image of structure of zinc coating. On Figure 3 there are cross-sections from all tested samples in various magnifications. There is seen the gaps between steel surface and the first zinc phase layer. The differences between morphology of zinc coating especially close to interface zinc/steel are evident. On sample 1 the coating is characterized by many cracks and small pores (bubbles) with very typical round shape. The diameter of these "bubbles" decreases with increased distance from steel surface. On other samples 2 and 3 the zinc coating layers have also cracks, but in small numbers, and no pores. 
Table 1 Chemical composition of steel (wt. \%)

\begin{tabular}{|c|c|c|c|c|}
\hline sample & Al & Si & P & Mn \\
\hline 1 & 0.10 & 0.06 & 0.02 & 0.33 \\
\hline 2 & 0.06 & 0.05 & 0.01 & 0.38 \\
\hline 3 & 0.06 & 0.06 & 0.01 & 0.67 \\
\hline
\end{tabular}
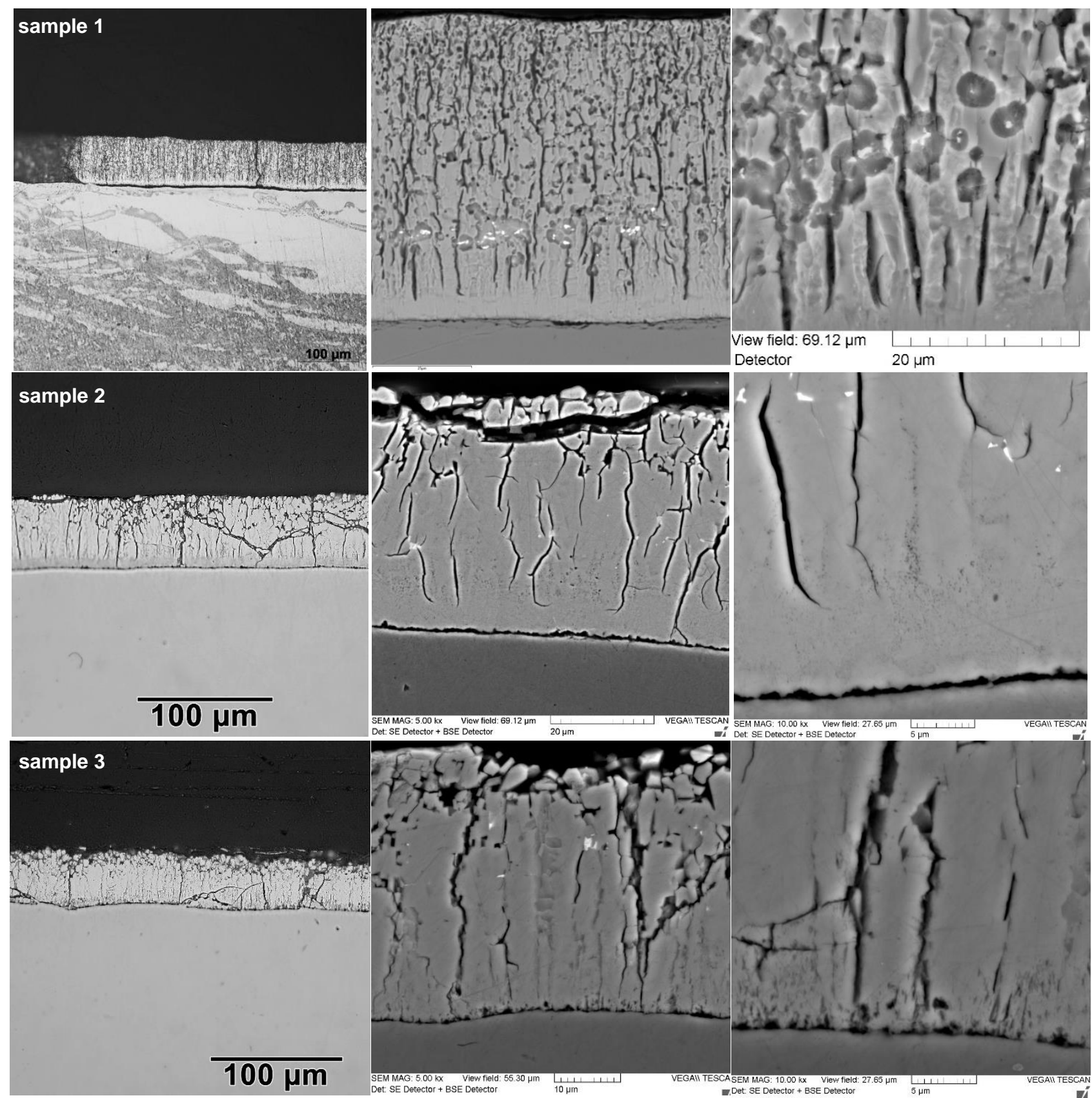

Figure $\mathbf{3}$ The structure of zinc coating on different samples

\subsection{Duplex coating of large panels}

Steel panels with dimension $950 \times 1770 \times 4.5 \mathrm{~mm}$, ca $230 \mathrm{pcs}$, were galvanized and then the powder coating was applied. Immediately a lot of defects as pinholes with various diameter (ca $5 \mathrm{~mm}$ ) occurred; some of them 
were up the coating surface and some in the coating (Figure 4). These bubbles form tiny craters on the surface of the coating. According to EN ISO 4628-2 the defects occurrence can be evaluated as 2-2(S2) to 2-2(S3), i.e. ca 0.05 to $0.5 \%$ from total surface of panel. Thickness of zinc coating was ca $75 \mu \mathrm{m}$ and powder coating ca $230 \mu \mathrm{m}$.

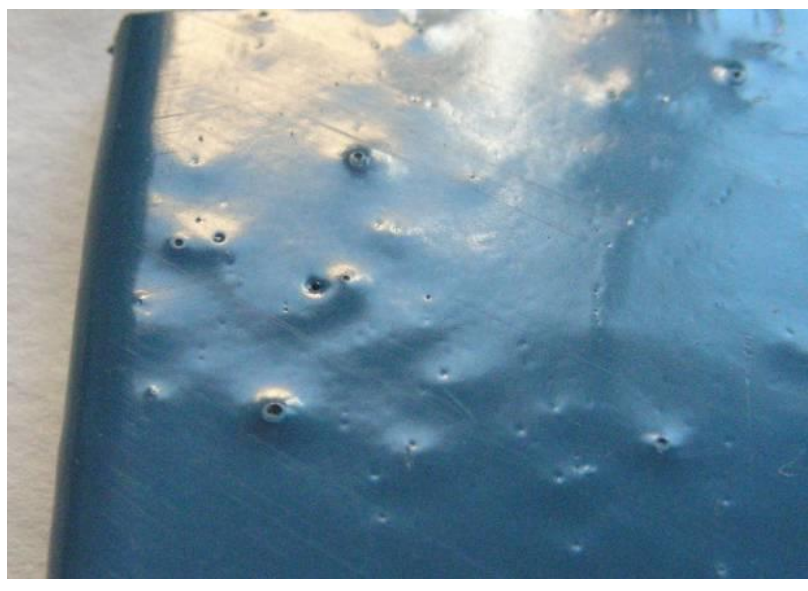

Figure 4 Example of defects on panels - pinholes

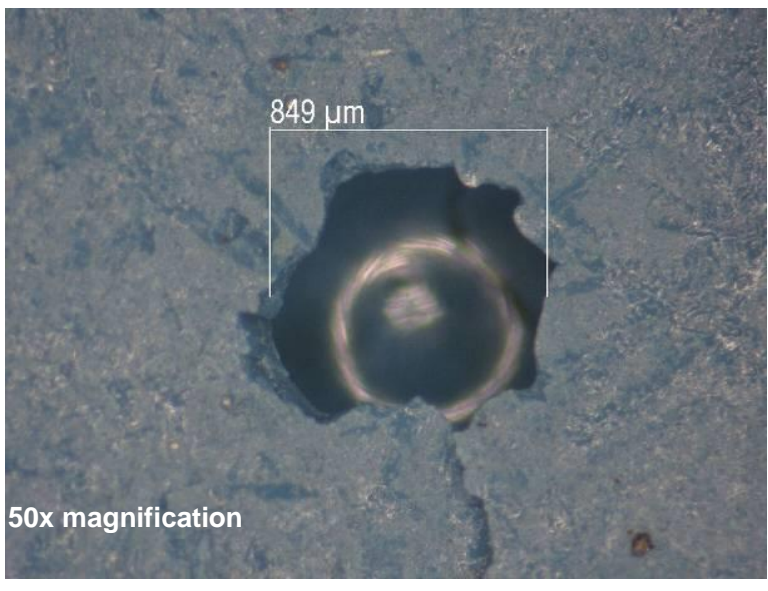

Figure 5 Defect on interface between zinc and organic coating

The microscopic evaluation shows large defects in the shape of bubbles on the interface between organic and zinc layers (Figure 5). The cross-sections of zinc coating illustrate the pores and cracks in the layer and especially on the interface of zinc coating and substrate steel (Figure 6).

The powder coating layer is cured by heating till ca $230^{\circ} \mathrm{C}$. In this temperature the recombination of diffused hydrogen in steel occurred and created such defects in zinc and subsequently in organic coating layers.

\section{CONCLUSION}

It is estimated that $70-80 \%$ of produced steel is minimally $1 \times$ pickled in acid during its technological treatment.

Microscopic examination was carried out on the various types of products which shown defects in coatings (galvanized, additional organic coatings) probably caused by hydrogen charging. It cannot be called embrittlement as the mechanical or corrosion properties of substrate steel were not affected, but the quality of surface treatments as corrosion protection was significantly negatively affected.

The hydrogen content in steels may have unfavorable effect on their physical, mechanical and electrochemical properties. Depending on their Si content steels in hot-dip galvanizing release the hydrogen absorbed during pickling in a different way [2]. The zinc coating grows evenly and quickly on category $C$ steel (samples $1-3$ of small artefacts). At the same time, hydrogen loss begins to occur immediately after the immersion of the steel into zinc bath. The emitting hydrogen causes a rapid removal of the $\mathrm{Fe} / \mathrm{Zn}$ alloy layers from the interface of steel/coating. Due to centrifuge galvanizing the only phase $\zeta$ without top phase $\eta$ is formed and coatings are more fragile in principle. On all samples galvanized by centrifuge technology the gaps between steel surface and zinc coating layer are evident. But only on sample 1 which had been pickled twice (once for mild scale removal, secondary as standard pretreatment before galvanizing) the pores (bubbles) formed in zinc layer.

The measurement of hydrogen content in steel is difficult due to very low levels of diffusible hydrogen present in them (cca 1-2 ppm) and the possibility of contamination from extraneous sources in the analysis. Hydrogen content in galvanized articles on the level of several ppm has no impact on the deterioration of steel mechanical properties [3], but he effect on the adhesion of zinc coating or forming the defect during subsequent powder coating of such articles may be more critical. 

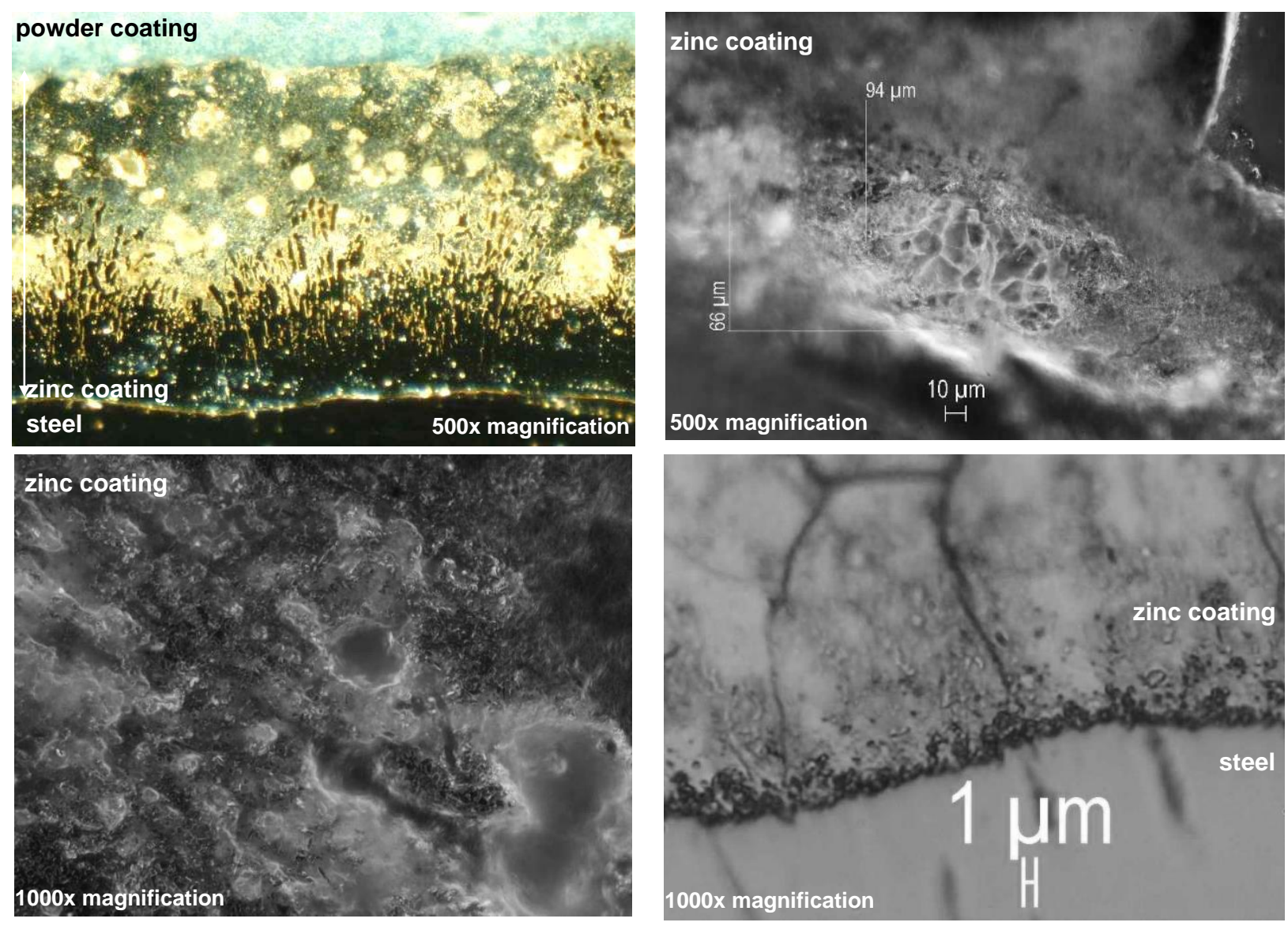

Figure 6 The cross-section of zinc coating with powder coating

Powder coating over galvanizing presents its own set of problems. There are few reasons of pinholes forming in powder coating - the distinct polyester resin particles that make contact with the surface of the galvanized steel do not fuse concurrently (the majority of cases), incomplete curing process, not proper pre-treatment of galvanized surface, etc. [4]. Pinholes develop from a process called "outgassing". Pinholes occur during the cure - as the part heats up, gasses that are trapped on or inside the part escape through the powder, causing holes or bubbles in the powder coating. The source of gasses may be surface contamination, moisture on surface, but the hydrogen charging of steel is also one of them. Atomic hydrogen, which penetrated into steel during pickling, only emits from it partly during the immersion in the zinc bath. A other amount in released from steel and zinc layer a few days after the galvanizing. Every next heating of steel accelerates this process, e.g. curing of powder coatings. During the curing the coatings re sintering and block free hydrogen release resulting into pinholes and craters in coating layer. Pre-heating the galvanized steel before painting is one of possibilities how to reduce the pinholes occurrence, but the temperature should be minimum $280^{\circ} \mathrm{C}$. During this temperature the diffusion of zinc phases occur, too, which may cause another problem with lower adhesion of zinc coating.

These two case studies show how important is detailed evaluation of failure by combination of methods for identification of reason of failure and make prevention means if it is possible (choice of steel category, each step in technology, parameters during galvanizing, etc.).

\section{ACKNOWLEDGEMENTS}

This paper had been done with funding of TA ČR project TH02020550 Research of the effect of chemical composition and metallurgical surface conditions of substrate steel onto quality of galvanized coating, 


\section{REFERENCES}

[1] BARRERA, O., BOMBAC, D., CHEN, Y., DAFF, T.D., GALINDO-NAVA, E., GONG, P., HALEY, D., HORTON, R., KATZAROV, I., KERMODE, J.R., LIVERANI, C., STOPHER, M., SWEENEY, F. Understanding and mitigating hydrogen embrittlement of steels: a review of experimental, modelling and design progress from atomistic to continuum, J. Mater. Sci. 2018, vol. 53, pp.6251-6290

[2] MAASSE, P., PEISSKER, P. Handbook of Hot-Dip galvanization. Wiley-VCH, Weinheim, 2011.

[3] SOZANSKA M., WEGRZYNKIEWICH, S., KIELBUS, A., MOSCICKI, A., HAJDUGA, M. Analysis of the hydrogen concentration in the products made of steel grade 41Cr4, Ochrona przed Korozja, 2017, vol. 60, no. 4, pp. 96-101

[4] DEAN, N. Powder Coating Over Galvanizing [online]. [viewed 2019-05-01] https://www.powdercoatingonline.com/ powder-coating-over-galvanizing/ 